\title{
Allocation of echo integrator output to small larval insect (Chaoborus sp.) and medium-sized (juvenile fish) targets
}

\author{
Reiner Eckmann * \\ Institute of Freshwater and Finh Ecology, Berlin. Germamy
}

\begin{abstract}
In acoustic sampling for fish, thresholding is normally applied to eliminate the unwanted contribution of noise to the integrator output. Since thresholding discriminates against small targets, this technique cannot be used for the quantitative study of these small targets in the presence of larger ones. When the integrator output (area backscattering coefficient, $s_{\mathrm{a}}$ ) due to one size class of targets is plotted vs. decreasing $s_{1}$-threshold (volume backscattering strength) in $1 \mathrm{~dB}$ steps, the resulting curve can be described by an asymptotic Bertalanffy growth function. If the slope of the resulting curve decreases at some intermediate threshold level and then increases again before the final plateau is reached. the total integrator output can be allocated to two groups of targets according to the linearity principle in acoustics. The maximum $s_{\mathrm{a}}$ value for the larger targets ( $s_{\mathrm{a}}$-large) is estimated by fitting a Bertalanffy function to the lower part of the curve. The echo integral that corresponds to the smaller targets is then found by subtracting $s_{\mathrm{a}}$-large from the $s_{\mathrm{a}}$ value that is reached at the lower threshold level. In a shallow (max. depth $8.5 \mathrm{~m}$ ) mesotrophic lake of 24 ha surface area in northeastern Germany. this new technique was tested to estimate the abundance of insect (Chaoborus) larvae in spring. Hydroacoustic data were sampled during night, when juvenile roach occurred together with midge larvae in the pelagic zone. Chaoborus abundance was estimated by replicate vertical tows of a $1 \mathrm{~m}^{2}$ plankton $\mathrm{ncl}$ of $0.5 \mathrm{~mm}$ mesh size. The $s_{\text {al }}$ values that were allocated to Chaoborus larvae were significantly correlated to mean Chaoborus abundance. This relationship was then used to map the lakewide distribution of Chaoborus larvae on two dates in spring. (C) 1998 Elsevier Science B.V.
\end{abstract}

Keywords: Acoustic size discrimination: Chaoborus larvae; Juvenile roach: Integrator output: Use of Bertalanffy function

\section{Introduction}

The precision of abundance estimates of differently sized acoustic targets depends to a great extent on the reliable allocation of integrator output to the various size classes of targets. If an equal proportion of single targets is resolved for each size class.

* Limnologisches Institut. Universitït Konstanz, Postfach 5560. D-78457 Konstanz. Germany. Tel.: + 4907531 882828; fax: +4907531883533; e-mail: reiner.eckmann@uni-konstanz.de integrator values are usually allocated according to the frequency distribution of target strengths. In many cases, however, single target resolution decreases with decreasing target strength, and a different method must be adopted.

The simplest and widely used alternative is to consider the echoes from small targets as noise and to eliminate their contribution to the total echo integral by thresholding (MacLennan and Simmonds, 1992). There seems to be no generally accepted, objective way to choose the appropriate threshold 
level, so this is usually done rather subjectively according to the operator"s experience.

Since thresholding discriminates against small targets, this technique cannot be used to estimate the abundance of these small targets. To do this, their contribution to the overall echo integral must be quantified. This can be achieved under different scenarios.

First, the small targets that are of interest can only be insonified in the presence of larger targets, while these larger targets can also be insonified in the absence of the smaller ones. The echo integral due to the larger targets may be determined during a certain time of the day, and then subtracted from the integral due to the large plus small targets that is measured during a different time of the day. Second. both small and large targets cannot be insonified alone. because they always occur together in the only habitat that is accessible to hydroacoustics.

The second scenario is often found in shallow. meso- to eutrophic lakes of the northeastern lowlands in Germany. In these lakes, Chaoborus larvae are abundant. They escape from fish predation by living in the anoxic hypolimnion, mostly even hidden in the sediment. during the day. At dusk. they ascend into the epilimnion where they feed on zooplankton, and they descend again at dawn. In parallel, juvenile fish display a diel horizontal migration. invading the pelagic zone at dusk and returning to the littoral at dawn. In this situation, acoustic abundance estimates of either juvenile fish or Chaoborus larvae require that integrator values are allocated according to clearly defined, objective criteria.

This paper proposes a method for distributing integrator values between two clearly distincl size classes of targets. To illustrate potential applications of this method. the horizontal distribution of Chaoborus larvae in a shallow mesotrophic lake was analysed and is presented as contour maps.

\section{Materials and methods}

Kleiner Doellnsee is a small ( 24 ha surface area), shallow (max. depth $8.5 \mathrm{~m}$ ) lake of glacial origin situated in the northeastern lowlands in Germany about $50 \mathrm{~km}$ north of Berlin. The lake is mesotrophic with average summer concentrations of $20 \mu \mathrm{g} / 1$ total P and $10 \mu \mathrm{g} / \mathrm{l}$ chlorophyll-a (Eckmann, 1995). During winter and summer stagnation. the hypolimnion may become completely anoxic, and hydrogen sulphide is always present in the extremely soft bottom sediment. The lake's fish fauna of 10 species (Eckmann, 1995) is largely dominated by roach (Rutilus rutilus) and perch (Perca fluciatilis). During the growing season. the pelagic zone is almost completely clear of fish during the day, while at dusk it is invaded by young age classes of roach and perch. In parallel, Chaoborus sp. larvae ascend from the sediment into the oxygen-rich pelagic zone. At dawn, both fish and Chaoborus larvae are clear of the epilimnion (Eisenbeiss, 1995).

Phantom midge larvae were sampled with a fourwalled pyramidal plankton net of $1 \mathrm{~m}^{2}$ opening area and $500 \mu \mathrm{m}$ mesh size. Sampling took place during the night. when the echogram showed that the larvae had completed their upward migration. Only the upper $6 \mathrm{~m}$ of water were sampled. because during summer stagnation, the larvae always ascended to less than $6 \mathrm{~m}$ depth. Three replicate tows were made at each of three sampling sites. Additionally, three replicate tows from $2 \mathrm{~m}$ depth to the surface were made at each site. This procedure was adopted to estimate by subtraction the average abundance of Chaoborus larvae between 2 and $6 \mathrm{~m}$ depth, because hydroacoustic data could not be sampled in the uppermost $2 \mathrm{~m}$ of water. The three sites (west. centre, east) that were marked with buoys were sampled on six occasions during summer 1995. At each sampling site, the six vertical tows were made while the boat was drifting within an area of 10-20 $m$ diameter. If the boat did not drift passively, it was rowed to different spots between successive samplings. This avoided sampling the same body of water between successive hauls. If the boat drifted out of the sampling area. it was rowed back. After each vertical tow, the net was thoroughly washed using a battery-powered bilge pump, and the larvae were preserved in $5 \%$ formalin solution. Samples were sorted into large (4th instars, the last larval stage prior to pupation) and small specimens if necessary, and the whole sample was counted in each case.

Acoustic data were sampled with a SIMRAD EY500 scientific sounder with $70 \mathrm{kHz}$ split-beam transducer. The system was calibrated with a stan- 
dard copper sphere using software that is supplied by the manufacturer. Pulse duration was set to $0.2 \mathrm{~ms}$, handwidth to $7 \mathrm{kHz}$ and ping rate to $10 / \mathrm{s}$.

The transducer was mounted in a fin that was suspended from the side of the boat at about $0.5 \mathrm{~m}$ depth. Boat speed was around $1 \mathrm{~m} / \mathrm{s}$. To observe fin h and Chaoborus migrations at dusk, acoustic data were sampled along a standard transect of about 400 $m$ length that runs across the deepest part of the lake. After dusk migrations of fish and Chaoborus larvae were completed, acoustic data were sampled. The hout was steered at about $0.5 \mathrm{~m} / \mathrm{s}$ in a circle of whuut $10 \mathrm{~m}$ radius around each of the three buoys until at least 3000 pings had been collected. Immediately afterwards, Chaoborus samples were taken. To map the horizontal distribution of Chaoborus larvae. woustic data were sampled on two parallel transects wross the lake and on one transect along the longest aris of the lake.

During postprocessing with EP500 software. time-varied gain was set to $20 \log R$. The integration threshold was decreased in $1-\mathrm{dB}$ stcps from -30 down to $-60 \mathrm{~dB}$ and then in $2-$ or $5-\mathrm{dB}$ steps down to $-75 \mathrm{~dB}$. Sample units were subdivided so that cach subunit contained approximately 1000 pings. For each subunit, the area backscattering coefficient ,$\left[\mathrm{m}^{2} / \mathrm{ha}\right]$ was plotted versus integrator threshold.

\section{Results}

Chaoborus abundance varied both bctwcen sampling dates and between sites during summer 1995 (Table 1). On four out of six occasions, the highest abundance was observed at the central site, and on five occasions the lowest abundance occurred at the wcstern site. Average abundance per unit surface area (from 2 to $6 \mathrm{~m}$ depth) ranged from 45 to 1056

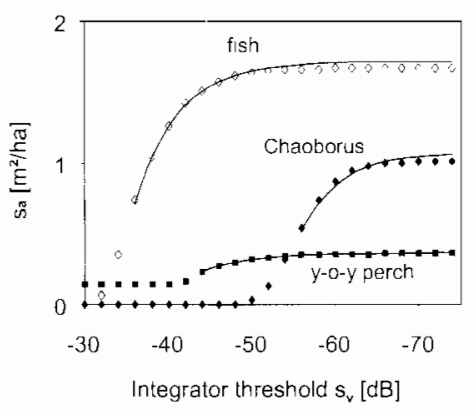

Fig. 1. Integrator output (area backscattering coefficient in $\mathrm{m}^{2} /$ ha) as a function of integrator threshold (volume backscattering strength in $\mathrm{dB}$ ) for three size classes of targets. Data were sampled when each of the three classes of targets could be insonified separately.

ind. $/ \mathrm{m}^{2}$. Standard errors ranged from 4 to $60 \%$ of the mean and were negatively correlated with average abundance $(p<0.01)$. During the first two samplings in May, only large (10.6 $\pm 0.9 \mathrm{~mm}$ length) Chaoborus larvae were present, while a new generation of larvae appeared in June. These specimens contributed between 2 and $17 \%$ to the total number of larvae in June. In the September samples, large and small larvae could still be differentiated easily, while in the October sample, all larvae were of similar size but significantly smaller $(8.7 \pm 1.3 \mathrm{~mm})$ than in May $(p<0.01)$.

The evening ascent of Chaoborus larvae from the lake bottom occurred approximately at the same timc as when fish started to move from the littoral into the pelagic zone. These fish were mainly roach of age classes 1 to 3 and perch of age class 1 (Eisenbeiss. 1995). Fish and Chaoborus migrations lasted for about 30 to $60 \mathrm{~min}$, and this was the only time when both groups of targets could be separately analysed acoustically. During darkness, both Chaoborus and fish always inhabited the same body of water until they returned to their daytime habitats at dawn.

Table 1

Mean abundance (individuals $/ \mathrm{m}^{2}$ ) \pm I SD of Chaoborus spp. larvae between 2 and $6 \mathrm{~m}$ depth at three sampling sites in Kleiner Docllnsec during summer 1995

\begin{tabular}{lllrrrr}
\hline & 10 May & 17 May & 15 June & 19 June & 4 Sept. \\
\hline West & $171 \pm 27$ & $149 \pm 34$ & $45 \pm 27$ & $149 \pm 62$ & $934 \pm 71$ & $244 \pm 59$ \\
Centre & $224 \pm 48$ & $665 \pm 191$ & $525 \pm 40$ & $814 \pm 43$ & $1056 \pm 59$ & $488 \pm 55$ \\
East & $465 \pm 18$ & $153 \pm 21$ & $167 \pm 24$ & $448 \pm 81$ & $170 \pm 39$ & $739 \pm 56$ \\
\hline
\end{tabular}

Vilues are based on the difference between three replicate tows from $6 \mathrm{~m}$ depth to the surface and from $2 \mathrm{~m}$ depth to the surface. 

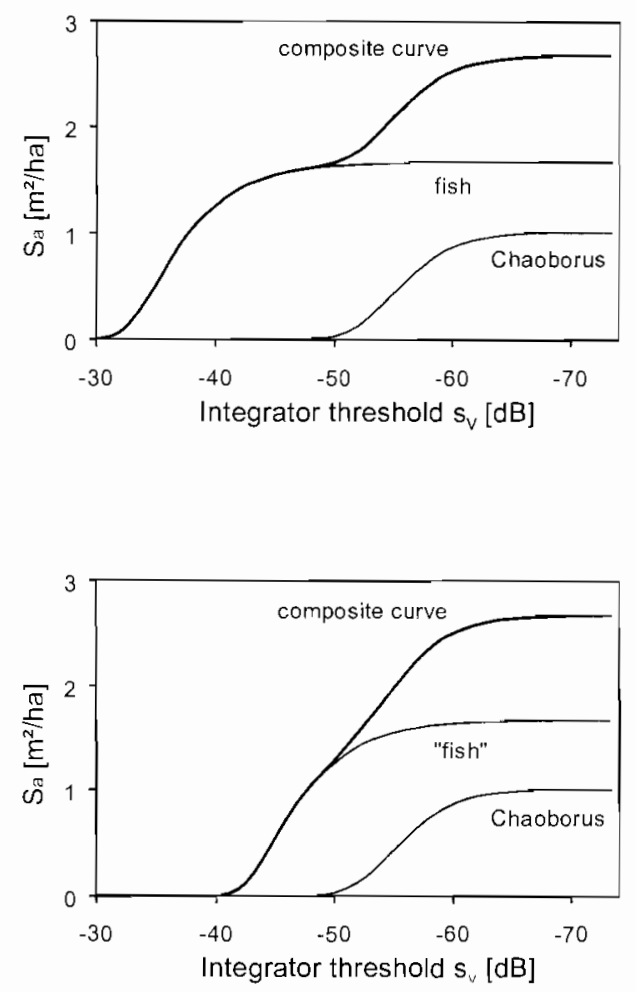

Fig. 2. Integrator-threshold curves for large Chaobortus larvae (original $s_{a}$ values multiplied by 5 ) and fish. The composite curve is the linear addition of the $s_{a}$ values. In the lower panel, the integrator-threshold curve for fish is shifted to a lower threshold by $10 \mathrm{~dB}$.

Typical integrator-threshold values for large Chaoborus larvae, young-of-the-year perch and for juvenile fish are shown in Fig. 1. These curves can be described by Bertalanffy functions, which are otherwise normally used to describe the asymptotic growth of fish. For the asymptotic upper part of the curves, the deviation between measured and calculated values is of the order 1 to $5 \%$.

When Chaoborus larvae and juvenile fish were insonified simultaneously, the integrator-threshold curves were characterised by decreasing slopes at intermediate threshold levels, until the final plateau was reached. These curves are interpreted as the result of a simple addition of the integrator outputs due to juvenile fish and due to Chaoborus larvae. This situation is shown in Fig. 2 (upper panel). In this particular case, the integrator output due to fish has almost reached its maximum value when the integrator output due to Chaoborus larvae just starts to build up at about $-48 \mathrm{~dB}$ threshold level. When a Bertalanffy function is fitted to the composite curve for $x$-values from -36 to $-48 \mathrm{~dB}$. the asymptotic $s_{\text {a }}$ value due to fish is estimated at 1.71 , which is $102 \%$ of the true maximum $s_{a}$ value due to fish.

If the integrator-threshold curve due to fish is shifted to lower threshold levels, the intermediate plateau of the composite curve becomes less pronounced (Fig. 2, lower panel). In this situation, only a smaller number of data points may be used to fit a Bertalanffy function to the lower. left-handed part of the composite curve. The range of data points to be included in the calculation was defined by that part of the composite curve for which the slope is continuously decreasing. In this way, the lower part of the curve that may have increasing or constant slope is discarded. as well as the central part of the curve, where the slope starts to increase again. In the example given in Fig. 2 (iower panel), this means that only 3 data points are available for fitting a Bertalanffy funetion. Hence. the $s_{a}$ value due to fish could not be estimated reliably. This situation was observed during summer and autumn, when the integrator-threshold curves did not show an intermedi-

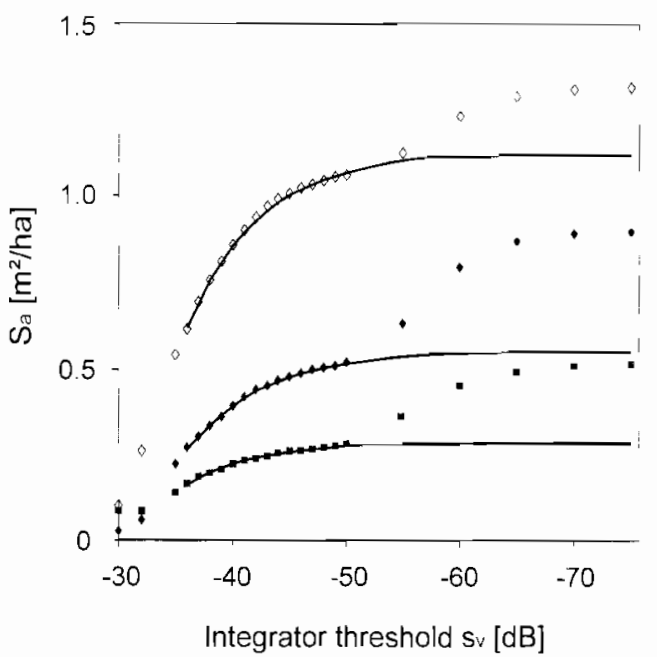

Fig. 3. Integrator-threshold curves for three subtransects on 11 May 1995 in Kleiner Doellnsee. Bertalanffy functions wcre fitted to the left-hand parts of the curves where slopes are continuously decreasing. The asymptotic function value is an estimate of the integrator output due to the larger targets. 
its depression. but increased steadily to the final : lateatl. During this time, in addition to juvenile fish and Chaoborus larvae, the open water was inhabited n! loung-of-the-year perch and possibly by young1t-the-year roach (Radke, pers. commun.). Adding ntegrator-threshold curves that are characteristic for unile lish. young-of-the-year fish and Chaoborus iariae will eventually suppress the intermediate plalealu

During May, however, when the pelagic zone was ?ianly inhabited by large Chaoborus larvae and . nulle fish, it was possibie to estimate $s_{\text {a values }}$ and to fish (Fig. 3). Since each of the six circular anutic transects sampled at the buoy stations was $\therefore$ mlsed in 3 to 7 subunits of approximately 1000 ?III- the standard deviation of the fish $s_{\text {at }}$ values - iuld be calculated for each transecl. These ranged rom 16 to $63 \%$ of the mean. For each subtransect. ine difference between a fish $s_{\text {id }}$ value and the final maximal $s_{31}$ value was allocated to the Chaoborus $s_{i 1}$ valuc. The standard deviations of the Chaoborus $s_{\text {is }}$ walues for each transect ranged from $41013 \%$ of the mean. Finally, a relationship between mean
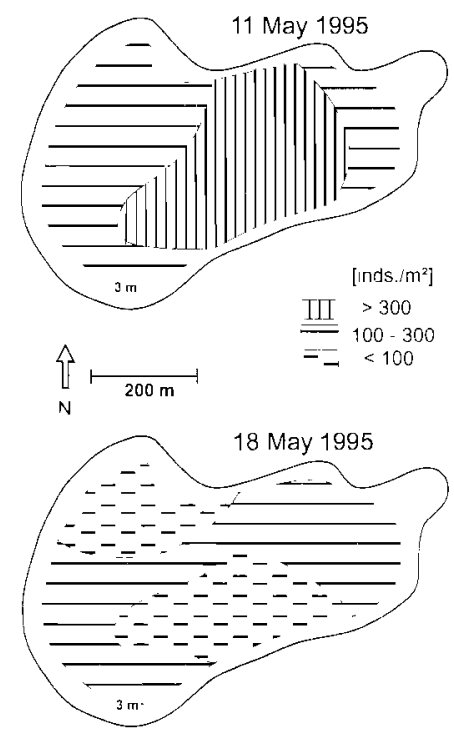

Fig. +. Isopleths of Chaoborus abundance from 2 to $6 \mathrm{~m}$ depth in the pelagic zone (where the depth is $>3 \mathrm{~m}$ deep) of Kleiner Donllnsee on two dates in May 1995. Data wore sampled on two $\therefore$ ansects across the lake and one transect along its longest axis. and they were analysed in subtransects of about 1000 pings each teyurvalent to about $100 \mathrm{~m}$ length). Isolines werc drawn by hand.
Chaoborus $s_{\mathrm{a}}$ values per transect and the mean Chaoborus abundance from net samples was established as $y=-255+188629 x\left(n=6 . r^{2}=0.91\right)$, where $y$ is Chaoborus abundance in individuals $/ \mathrm{m}^{2}$ and $x$ is Chaoborus $s_{\text {a }}$ values in $\mathrm{m}^{2} /$ ha.

Based upon this relationship, the lakewide horizontal distribution of Chaoborus larvae was mapped on May 11 and May 18, 1995. The near-shore region down to $3 \mathrm{~m}$ deep was excluded from this analysis because of the presence of macrophytes and of large groups of fish in the shallow areas. For each subtransect that comprised about 1000 pings, a Chaoborus $s_{\text {¿t }}$ value was estimated, and the corresponding abundance level was calculated. Finally, isopleths of different abundance levels were drawn by hand (Fig. 4).

The abundance of Chaoborus was not homogeneously distributed throughout the lake on these two occasions. The horizontal distribution patterns were also dissimilar. The average Chaoborus densities, calculated from unweighted subtransect means, decreased from 300 to 140 ind./ $\mathrm{m}^{2}$ within one week. During this time, dense clouds of emerging adults could be seen above the lake surface at night.

\section{Discussion}

Based upon the linearity principle in acoustics (MacLennan and Simmonds, 1992), the integrator output of a scientific echosounder can be conceived as the linear addition of the echo energy from all targets that are insonified simultaneously. Therefore, it is theoretically possible to redistribute the integrator output to the different size classes of targets such as the frequency distribution of target strengths. The advantage of this is that it allows the allocation of integrator outputs to small and to distinctly large targets, even if single-target resolution is nil.

Chaoborus larvae could only be insonified separately during a short time interval when they emerged from the sediment at dusk. Even in this situation, single-target resolution was low and only a few targets were observed in the size range of -72 to $-75 \mathrm{~dB}$. This may be caused by the high density of larvae (several hundred larvae per one or two $\mathrm{m}^{3}$ ). and by their backscattering characteristics. Chaoborus larvae possess two pairs of kidney-shaped tracheal bladders that are 6-8 $\mathrm{mm}$ apart. Phase 
stability in the backscattered sound wave may, therefore, be weak, and the echo eventually rejected according to the single-target criteria implemented in the EY500 sounder.

Irrespective of single target resolution, the proposed method permits the estimation of abundance of small targets instead of considering them as noise. Their identity must, of course, be verified by traditional sampling techniques. Then a relationship between integrator output due to small targets and their abundance can be established. The main disadvantage of this method is that it is only applicable to targets that are both simultaneously insonified and of clearly distinct sizes. If the integrator-threshold curves overlap too much. the $s_{\mathrm{a}}$ value due to the larger targets cannot be estimated. This was the case in Kleiner Doellnsee from early June until October. Nevertheless, the use of the integrator output as a function of integration threshold is a valuable tool that will focus the operator's attention on the problem of selecting an appropriate threshold level. In the situation illustrated in Fig. 2 (lower panel), small changes in threshold level can result in considerable changes of integrator output.

This method of data analysis may be equally useful for abundance estimates of large targets in the presence of small targets. Normally, an integrator threshold level is selected subjectively by progressively increasing the threshold until almost all noise disappears from the echogram. The error that is now associated with the corresponding abundance estimate is largely unknown. It is less subjective to choose a threshold level by inspecting integratorthreshold curves, and the best alternative is to estimate $s_{a}$ values from those curves by fitting Bertalanffy functions. The estimated $s_{a}$ value for the larger targets is distributed among these targets according to the frequency distribution of target strengths.

Phantom midge larvae may play an important role in the pelagic food web of eutrophic lakes, where their impact on the zooplankton community via topdown control by an invertebrate zooplanktivore is of special interest within the framework of biomanipulation (Benndorf et al., 1988: Benndorf, 1990; Elser et al., 1987: Yan et al.. 1991). Therefore, precise knowledge of Chaoborus abundance and distribution patterns is often required. Without mapping the lakewide distribution of the larvae. their overall decrease by about $50 \%$ between May 11-18 might have been undetected. The analysis of distribution patterns also helps to assess how representative is one particular sampling station for the whole system.

Even when Chaoborus abundance cannot be mapped throughout the growing season by the method proposed here. it might still be useful to apply it on certain dates. The horizontal heterogeneity of Chaoborus distribution will become evident and, assuming it to be representative for the whole period. the sampling protocol adjusted accordingly.

The Chaoborus $s_{i 1}$ values were very similar between the subtransects sampled at the buoy stations, with coefficients of variation of only $4-13 \%$ when using an averaging interval of about $50 \mathrm{~m}$. In contrast, the fish $s_{\text {a }}$ values had much wider coefficients of variation of $16-63 \%$ for the same subtransects. This suggests that the fish distribution at the same spatial scale is much more patchy than that of Chaoborus. However, on a finer spatial scale. the Chaobortis can be seen to be patchily distributed. This is clearly seen in the results from the net samples (Table 1) where coefficients of variation ranged from 4 to $60 \%$. On a broader scale of $\sim 100$ $\mathrm{m}$ along the transects across the lake. Chaoborus $s_{\text {it }}$ values were also less variable than those for fish. In seven subtransects sampled along the longest axis of the lake, fish $s_{i 1}$ values differed by a factor of 4.6. while those for Chaoborus differed by only a factor of 2.1. These data suggest that there is a very fine-scaled patchiness in the Chaoborus distribution that makes it difficult 10 sample the larvae representatively by standard techniques in a reasonable amount of time. Averaging acoustic data along short. circular transects is proposed as a tool to overcome this difficulty.

\section{Conclusions}

Plots of the integrator output (area backscattering coefficient in $\mathrm{m}^{2} / \mathrm{ha}$ ) from a scientific echosounder vs. integrator threshold (volume backscattering strength in $\mathrm{dB}$ ) can be described by Bertalanffy functions (Fig. 1).

If the integrator outpul is based on the echoes from two clearly distinct size classes of targets, these 
$\because \therefore$ are characterised by decreasing slopes at interandate threshold levels (Fig. 2). The asymptote mevrator output due to the larger targets may then i: found by fitting a Bertalanffy function, and the - ntribution of the smaller size class can be deter- ined by subtraction (Fig. 3).

B! calibration of the area backscattering coeffi- Rent against conventional abundance measuremint . the areal density of the smaller targets can be $\therefore$ aluuted acoustically. even when in the presence of $\therefore$.rer turgets. It is then possible to map the horizon$\therefore$ Jivibution of small targets like Chaoborus lar$\therefore$ (Flg, t).

Fitting Bertalanffy functions to integrator:.r. ?...te for the larger targets as compared to conveniinnal thresholding.

This method cannot be used when the two target sulup are insufficiently different in size, or if more than tho size classes are insonified simultaneously.

\section{Acknowledgements}

Christian Heims, Totok Hestirianoto, Karin Kleibs .nd Darid Ritterbusch helped during data acquisi: ion. counting of Chaoborus samples and processing acoustic data. The valuable collaboration of Cornelia Batussek is particularly acknowledged during the early stages of the study when the basic concept was being developed.

\section{References}

Benndorf. J., Schultz. H.. Benndorf, A., Unger, R., Penz, E. Kneschke. H.. Kossatz. K.. Dumke, R.. Hornig, W.. Kruspe, R.. Rcichel. S., 1988. Food-web manipulation by enhancement of piscivorous fish stocks: long-term effects in the hypertrophic Bautzen rescrvoir. Limnologia 19.97-110.

Benndorf. J.. 1990. Conditions for effective biomanipulation: conclusions derived fron whole-lake experiments in Europe. Hydrobiologia 200-201. 187-203.

Eckmann. R.. 1995. Fish species richness in lakes of northeastern lowlands in Germany. Ecol. Freshwater Fish 4. 62-69.

Eisenbeiss, D.. 1995. Untersuchungen $7 u$ den diurnalen Verteilungsmustern juveniler Plotzen (Rutilus rutilus (L.)) im Kleiner Doellnsee. Diploma Thesis. Univ. Freiburg. 55 pp.

Elser. M.M. von Ende. C.E., Sorrano. P.S.. Carpenter. S.R.. 1987. Chaoborus populations: response to food web manipulations and potential effects on zooplankton communities. Can. J. Zool. 65. 2846-2852.

MacLennan. D.N.. Simmonds, E.J., 1992. Fisheries acoustics. Chapman \& Hall. London. 325 pp.

Yan. N.D.. Keller. W., MacIsaac. H.J.. McEachern. L.J.. 1991 Regulation of rooplankton community structure of an acidified lake by Chaoborts. Ecol. Applications 1. 52-65. 